\title{
Postoperative serum $\alpha$-fetoprotein level is a useful predictor of recurrence after hepatectomy for hepatocellular carcinoma
}

\author{
DAISUKE NOBUOKA ${ }^{1,2}$, YUICHIRO KATO ${ }^{2}$, NAOTO GOTOHDA ${ }^{2}$, SHINICHIRO TAKAHASHI $^{2}$, \\ TOSHIO NAKAGOHRI ${ }^{2}$, MASARU KONISHI ${ }^{2}$, TAIRA KINOSHITA ${ }^{2}$ and TETSUYA NAKATSURA $^{1}$ \\ ${ }^{1}$ Section for Cancer Immunotherapy, Investigative Treatment Division, Research Center for Innovative Oncology and \\ ${ }^{2}$ Department of Surgery, National Cancer Center Hospital East, 6-5-1 Kashiwanoha, Kashiwa, Chiba 277-8577, Japan
}

Received December 18, 2009; Accepted March 3, 2010

DOI: $10.3892 / o r \_00000888$

\begin{abstract}
We evaluated the clinical value of perioperative $\alpha$-fetoprotein (AFP) and des- $\gamma$-carboxy prothrombin (DCP) levels in predicting recurrence of hepatocellular carcinoma (HCC) after curative resection, with a focus on the time course as surveillance tools. A total of 165 consecutive HCC patients who had undergone curative hepatectomy at our institution from 2005 to 2007 and whose serum AFP and DCP had been measured before and after hepatectomy were included in this study. The minimum postoperative levels within a 4-month period were used for analysis. Among the patients with a positive level of AFP before operation, the number of patients whose AFP level did not change from positive to negative after operation in the group with recurrence exceeded that in the group without recurrence $(48 / 60$, $80.0 \%$ vs. $4 / 23,17.4 \%$ ), and the difference was significant $(\mathrm{P}<0.001)$. Minimum postoperative AFP level was found to be a significant independent risk factor for recurrence by multivariate analysis $(\mathrm{P}<0.001)$. There was no statistically significant correlation between AFP level and grade of hepatitis activity $(\mathrm{P}=0.599)$. Postoperative AFP level is a
\end{abstract}

Correspondence to: Dr Tetsuya Nakatsura, Section for Cancer Immunotherapy, Investigative Treatment Division, Research Center for Innovative Oncology, National Cancer Center Hospital East, 6-5-1 Kashiwanoha, Kashiwa, Chiba 277-8577, Japan

E-mail: tnakatsu@east.ncc.go.jp

Abbreviations: AFP, $\alpha$-fetoprotein; DCP, des- $\gamma$-carboxy prothrombin; HCC, hepatocellular carcinoma; CT, computed tomography; MRI, magnetic resonance imaging; US, ultrasonography; TMN, tumor-node-metastasis; UICC, International Union against Cancer; PPV, positive predictive value; NPV, negative predictive value; ALT, alanine aminotransferase; AFP-L3, Lens culinaris agglutinin-reactive $\alpha$-fetoprotein; GPC3, glypican-3; $\mathrm{HBsAg}$, hepatitis B s antigen; anti-HCV, anti hepatitis C virus antibody; AST, aspartate aminotransferase; T. Bil, total bilirubin; Alb, albumin; ChE, cholinesterase; PLT, platelet; PT, prothrombin time; ICG-R15, indocyanine green-retention at $15 \mathrm{~min}$

Key words: hepatocellular carcinoma recurrence, $\alpha$-fetoprotein, Des- $\gamma$-carboxy prothrombin, tumor marker, hepatectomy useful tool for predicting recurrence after curative hepatectomy. A positive level of AFP after operation might suggest a site of residual viable cancer. The need for effective adjuvant therapy and close follow-up is suggested in patients with a positive postoperative AFP level.

\section{Introduction}

Primary liver cancer, which consists predominantly of hepatocellular carcinoma (HCC), is the fifth most common cancer worldwide and the third most common cause of cancer mortality, and is becoming more prevalent not only in South-East Asia and Africa but also in Western countries (1-4). Therefore, great interest in HCC has recently developed all over the world.

Surgical resection is the most effective treatment for curable HCC. Although the short-term prognosis of HCC patients has improved due to advances in surgical technique and perioperative management, the long-term prognosis remains far from satisfactory due to frequent recurrence because of not only metastasis from the primary tumor but also multicentric carcinogenesis based on underlying hepatitis or cirrhosis. Therefore, control of recurrent disease is a major challenge in HCC treatment and, especially, the establishment of effective adjuvant therapy to prevent recurrence is required. However, there is no universal consensus at present (5). Secondly, because the outcome in cases of HCC recurrence is likely to be improved by early detection, postoperative surveillance is also required.

Serum levels of $\alpha$-fetoprotein (AFP) and des- $\gamma$-carboxy prothrombin (DCP) are well known as tumor markers of HCC (6). Although many study groups have indicated that preoperative levels of these tumor markers were prognostic factors for HCC (7-11), the clinical usefulness of postoperative levels for early detection of HCC recurrence remains unclear.

This study evaluated the clinical value of perioperative serum AFP and DCP levels in predicting HCC recurrence after curative resection, with a focus on the time course as surveillance tools.

\section{Materials and methods}

Patient selection. From January 2005 to December 2007, 192 consecutive patients with $\mathrm{HCC}$ underwent hepatectomy at the 
Table I. Baseline characteristics of study patients $(n=165)$.

\begin{tabular}{|c|c|}
\hline Age (years) & $67.2 \pm 8.6$ \\
\hline Gender (male/female) & $138(83.6) / 27(16.4)$ \\
\hline Viral infection [HBsAg(+)/anti-HCV(+)/both(+)/both(-)] & $30(18.2) / 97(58.8) / 6(3.6) / 32(19.4)$ \\
\hline Diabetes (present/absent) & $44(26.7) / 121(73.3)$ \\
\hline Tumor occurrence (initial/recurrent) & $108(65.5) / 57(34.5)$ \\
\hline Child-Pugh classification (Class A/B/C) & $150(90.9) / 15(9.1) / 0(0.0)$ \\
\hline AST (IU/l) & $56.2 \pm 38.1$ \\
\hline ALT (IU/l) & $57.2 \pm 44.6$ \\
\hline T. Bil (mg/dl) & $0.9 \pm 0.3$ \\
\hline $\operatorname{Alb}(g / d l)$ & $3.9 \pm 0.3$ \\
\hline $\mathrm{ChE}(\mathrm{IU} / \mathrm{l})$ & $247.0 \pm 71.0$ \\
\hline $\operatorname{PLT}\left(\mathrm{x} 10^{3} / \mathrm{mm}^{3}\right)$ & $15.9 \pm 16.9$ \\
\hline $\mathrm{PT}(\%)$ & $78.7 \pm 10.9$ \\
\hline ICG-R15 (\%) & $17.2 \pm 10.4$ \\
\hline Preoperative AFP (ng/ml) & $1464.0 \pm 6038.2$ \\
\hline Minimum postoperative AFP (ng/ml) & $695.7 \pm 7755.1$ \\
\hline Preoperative DCP (mAU/ml) & $1539.8 \pm 6204.2$ \\
\hline Minimum postoperative DCP (mAU/ml) & $39.9 \pm 125.3$ \\
\hline Tumor size $(\mathrm{cm})$ & $3.9 \pm 2.9$ \\
\hline No. of nodules (solitary/multiple) & $108(65.5) / 57(34.5)$ \\
\hline Histological differentiation (well/moderate/poor) & $29(17.6) / 92(55.8) / 44(26.7)$ \\
\hline Macroscopic vascular invasion (present/absent) & $7(4.2) / 158(95.8)$ \\
\hline Microscopic vascular invasion (present/absent) & $43(26.1) / 122(73.9)$ \\
\hline Intrahepatic metastasis (present/absent) & $14(8.5) / 151(91.5)$ \\
\hline Stage (UICC) (I/II/III/IV) & $86(52.1) / 62(37.6) / 15(9.1) / 2(1.2)$ \\
\hline \multicolumn{2}{|l|}{ Operative procedure } \\
\hline (trisegmentectomy/central bisegmentectomy/ & $2(1.2) / 2(1.2) /$ \\
\hline hemihepatectomy/segmentectomy/ & $18(10.9) / 15(9.1) /$ \\
\hline subsegmentectomy/partial resection) & $14(8.5) / 114(69.1)$ \\
\hline Surgical margin $(\mathrm{mm})$ & $2.7 \pm 4.2$ \\
\hline Histological findings of non-cancerous lesions (liver cirrhosis/ & $60(36.4) / 90(54.5) / 15(9.1)$ \\
\hline
\end{tabular}

Continuous variables are expressed as mean and standard deviation. Values in parentheses are percentages.

National Cancer Center Hospital East, Japan. Among them, 165 patients were enrolled in this study, excluding 14 who underwent non-curative resection, 9 who were lost to serial follow-up, and 4 who took oral warfarin, a DCP-inducing agent. Baseline characteristics of the patients are shown in Table I. None of the patients in this study received postoperative adjuvant therapy, including interferon.

Diagnosis of HCC. HCC was diagnosed using dynamic computed tomography (CT) or magnetic resonance imaging (MRI), considering hyper-attenuation in the arterial phase with washout in the late phase to indicate definite HCC (12) Intraoperatively, ultrasonography (US) was performed to determine whether other nodules were present in the liver or not (13). All nodules were confirmed to be HCC histopathologically after surgical resection. Pathological stage was assigned according to the tumor-node-metastasis (TNM) classification of the International Union against Cancer (UICC)
(14), and curative resection was defined as a negative surgical margin histopathologically. Tumor recurrence was defined as a newly developed lesion on CT or MRI.

Measurement of serum AFP and DCP concentrations. Serum AFP and DCP concentrations were determined within 1 month before operation and at least once within a 4-month period after operation, using a commercially available electrochemiluminescence immunoassay kit (Roche Co., Tokyo, Japan) and chemiluminescent enzyme immunoassay kit (Eisai Co., Tokyo, Japan), respectively. The minimum postoperative level was used for analysis, and the levels of the tumor markers before and after operation were compared. In this study, the cut-off levels for AFP and DCP were set as $10 \mathrm{ng} / \mathrm{ml}$ and $40 \mathrm{mAU} / \mathrm{ml}$, respectively.

Statistical analysis. Continuous variables were expressed as mean and standard deviation. The number of patients whose 
Table II. Sensitivity of $\alpha$-fetoprotein (AFP), des- $\gamma$-carboxy prothrombin (DCP) and combination of both.

\begin{tabular}{|c|c|c|c|c|c|c|}
\hline \multirow[b]{2}{*}{ Sensitivity before operation } & \multicolumn{2}{|c|}{$\begin{array}{c}\text { AFP }(\%) \\
\text { (Cut-off level; } 10 \mathrm{ng} / \mathrm{ml})\end{array}$} & \multicolumn{2}{|c|}{$\begin{array}{c}\text { DCP (\%) } \\
\text { (Cut-off level; } 40 \mathrm{mAU} / \mathrm{ml})\end{array}$} & \multicolumn{2}{|c|}{$\begin{array}{l}\text { Combination of } \\
\text { AFP and DCP }(\%)\end{array}$} \\
\hline & $83 / 165$ & 50.3 & $101 / 165$ & 61.2 & $130 / 165$ & 78.8 \\
\hline Sensitivity at recurrence & $63 / 114$ & 55.3 & $46 / 114$ & 40.4 & $82 / 114$ & 71.9 \\
\hline
\end{tabular}

tumor marker levels changed from positive to negative after operation was compared using $\chi^{2}$ test. Recurrence rates were analyzed according to the Kaplan-Meier estimate, and differences between subgroups were compared using the logrank test. Multivariate Cox's proportional hazard regression was used to determine the effect of all of the potential variables. Univariate regression analysis was used to evaluate the correlation between AFP level and grade of hepatitis activity. Kruskal-Wallis rank test was used to evaluate the correlation between AFP level and grade of underlying chronic liver disease. Mann-Whitney U test was used to evaluate the correlation between AFP level and recurrence. For all statistical tests, differences were considered significant at P-values $<0.05$. Data were analyzed with the statistical package, Dr. SPSS II ${ }^{\circledR}$ for Windows (SPSS Japan, Tokyo, Japan).

\section{Results}

Sensitivity of AFP and DCP. The sensitivity rates of preoperative AFP and DCP for detection of HCC were $50.3 \%$ $(83 / 165)$ and $61.2 \%(101 / 165)$, respectively. The combination of AFP and DCP increased the sensitivity to $78.8 \%(130 / 165)$ (Table II).

Until the end of follow-up, tumor recurrence was identified in 114 patients $(69.1 \%)$. Of these, $106(93.0 \%)$ had intrahepatic recurrence distant from the primary site, $3(2.6 \%)$ had local tumor recurrence and $13(11.4 \%)$ had extrahepatic recurrence (some patients had a first relapse at more than one site). The cumulative probability of overall recurrence was 46.2, 64.5 and $74.5 \%$ at 1,2 and 3 years, respectively. The sensitivity of AFP, DCP, and their combination at the time for detecting recurrence was $55.3 \%$ (63/114), $40.4 \%$ (46/114) and $71.9 \%$ (82/114), respectively (Table II).

The time course of AFP and DCP. Among the 83 patients with a positive AFP level before operation, the value decreased and changed to negative after curative operation in 31 patients $(37.3 \%)$, whereas it remained positive in $52(62.7 \%)$. On the other hand, among the 101 patients with a positive DCP level before operation, the value changed to negative after operation in $85(84.2 \%)$, whereas it remained positive in $16(15.8 \%)$. The rate of negative change of AFP was significantly lower than that of DCP $(\mathrm{P}<0.001)$.

Fig. 1 shows the changes in perioperative AFP level in patients with (Fig. 1A and B) and without (Fig. 1C and D) recurrence. Among the patients with a positive level of AFP before operation, the number of patients whose AFP level remained positive after operation in the group with recurrence exceeded that in the group without recurrence (48/60, $80.0 \%$ vs. $4 / 23,17.4 \%$ ) (Fig. $1 \mathrm{~A}$ and C), and the difference was

\section{A Recurrence (+)}

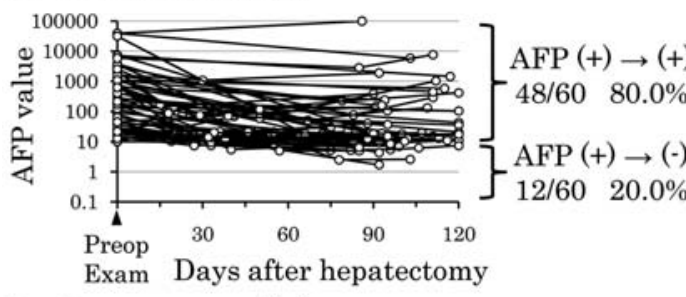

B Recurrence (+)

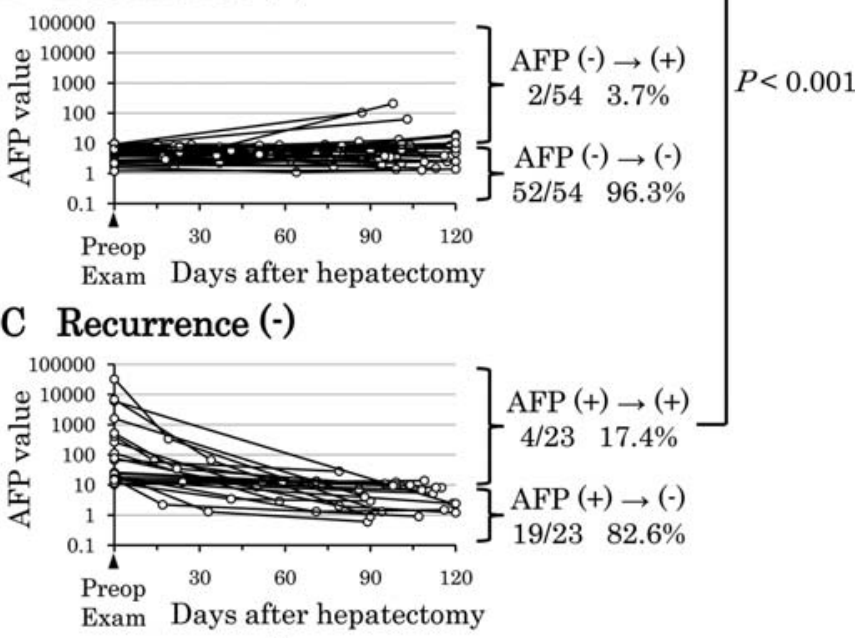

\section{Recurrence (-)}

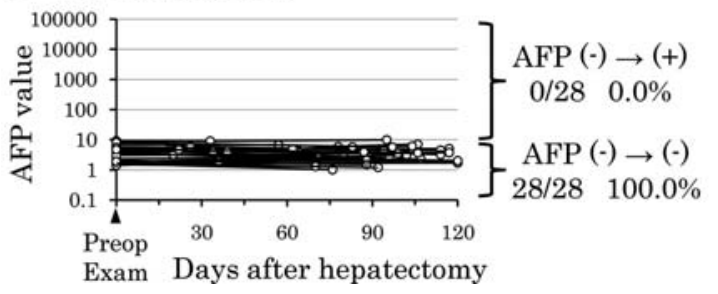

Figure 1. Pre- and postoperative serum $\alpha$-fetoprotein (AFP) level. (A) Patients with recurrence and positive AFP preoperatively. (B) Patients with recurrence and negative AFP preoperatively. (C) Patients without recurrence and positive AFP preoperatively. (D) Patients without recurrence and negative AFP preoperatively. Among the patients with a positive preoperative AFP level, the number of patients whose AFP level remained positive after operation in the group with recurrence exceeded that in the group without recurrence $(\mathrm{P}<0.001)$. Preop exam, preoperative examination.

significant $(\mathrm{P}<0.001)$. From a different viewpoint, among the 52 patients whose AFP level was positive both before and after operation, 48 (92.3\%) experienced recurrence later (Fig. 1A and C). Among the 82 patients whose AFP level was negative before operation, only $2(2.4 \%)$ had a positive AFP level after operation, and both of them $(2 / 2,100.0 \%)$ experienced recurrence (Fig. 1B and D). 


\section{A Recurrence (+)}

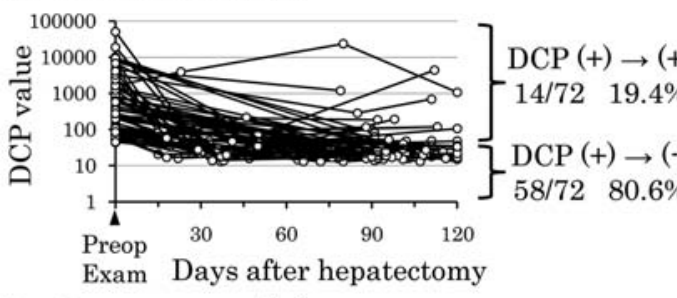

\section{B Recurrence (+)}

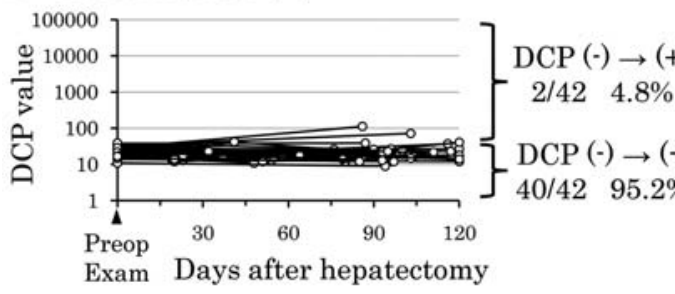

\section{Recurrence (-)}

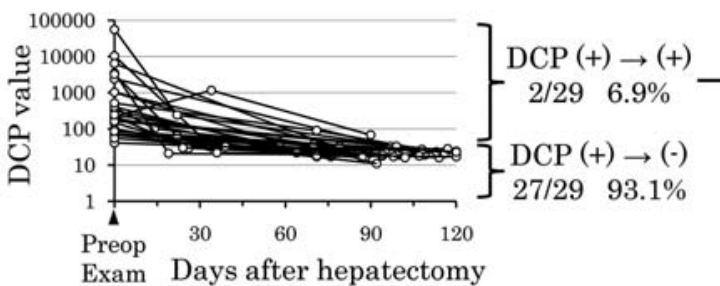

\section{Recurrence (-)}

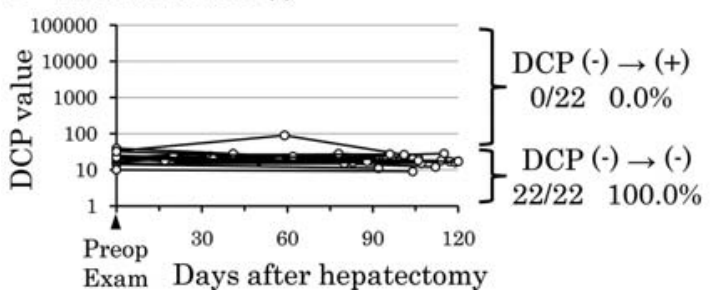

Figure 2. Pre- and postoperative serum des- $\gamma$-carboxy prothrombin (DCP) level. (A) Patients with recurrence and positive DCP preoperatively. (B) Patients with recurrence and negative DCP preoperatively. (C) Patients without recurrence and positive DCP preoperatively. (D) Patients without recurrence and negative DCP preoperatively. There was no significant difference in the number of patients whose DCP remained positive between the group with and without recurrence $(\mathrm{P}=0.118)$. Preop exam, preoperative examination.

Fig. 2 shows the changes in perioperative DCP level. In contrast to AFP, there was no significant difference in the number of patients whose DCP remained positive between the two groups $(14 / 72,19.4 \%$ vs. $2 / 29,6.9 \%$; $\mathrm{P}=0.118)$ (Fig. 2A and C). However, from a different viewpoint, among the 16 patients whose DCP level was positive both before and after operation, $14(87.5 \%)$ experienced recurrence later (Fig. 2A and C). Among the 64 patients whose DCP level was negative before operation, only $2(3.1 \%)$ had a positive DCP level after operation, and both of them $(2 / 2,100.0 \%)$ experienced recurrence (Fig. 2B and D).

Accuracy of minimum postoperative AFP and DCP. The sensitivity, specificity, positive predictive value (PPV), and negative predictive value (NPV) of minimum postoperative AFP, DCP and the combination of both for HCC recurrence were calculated. The sensitivity of minimum postoperative

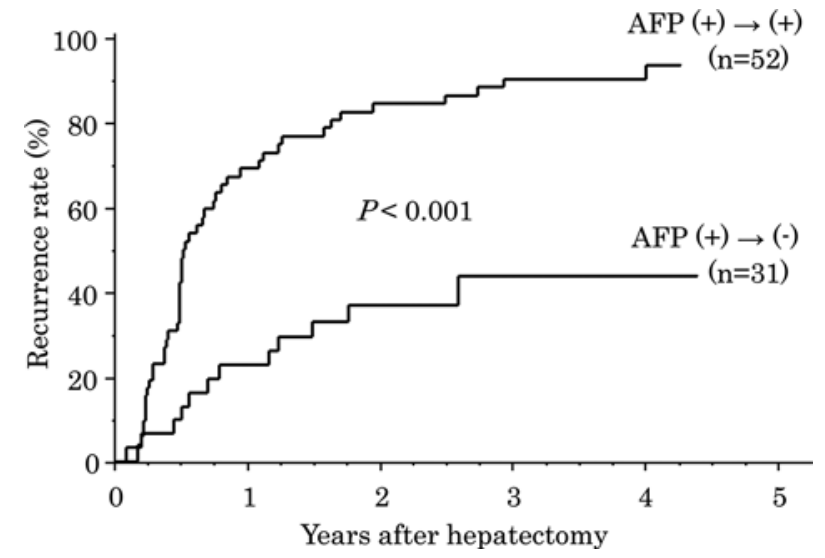

Figure 3. Overall cumulative recurrence rate curve according to minimum postoperative $\alpha$-fetoprotein (AFP) level. Recurrence rate was higher in the patients whose AFP level remained positive after operation than in those whose AFP level was changed from positive to negative $(\mathrm{P}<0.001)$.

AFP (43.9\%), DCP (14.0\%) and even the combination of both $(50.0 \%)$ was rather low. However, when classified by preoperative level, the minimum postoperative AFP level in patients with a positive preoperative level had higher sensitivity $(80.0 \%)$ while maintaining high specificity $(82.6 \%)$ and PPV (92.3\%) (Table III).

Univariate and multivariate analyses to identify the risk factors for HCC recurrence. The differences in cumulative recurrence rate of patients among the various risk factors stratified were evaluated by log-rank test. Age $(\mathrm{P}=0.031)$, tumor occurrence (initial/recurrent) $(\mathrm{P}<0.001)$, minimum postoperative AFP level $(\mathrm{P}<0.001)$, minimum postoperative DCP level $(\mathrm{P}=0.002)$, tumor size $(\mathrm{P}=0.026)$, number of nodules $(\mathrm{P}=0.007)$, microscopic vascular invasion $(\mathrm{P}<0.001)$, intrahepatic metastasis $(\mathrm{P}<0.001)$ and pathological stage $(\mathrm{P}<0.001)$ were found to be significantly related to recurrence, whereas preoperative levels of AFP $(\mathrm{P}=0.408)$ and $\mathrm{DCP}(\mathrm{P}=0.375)$ were not. Thereafter, multivariate analysis using Cox's proportional hazard model was performed to assess the independent importance of each variable studied. Tumor occurrence (initial/recurrent) $(\mathrm{P}<0.001)$, minimum postoperative AFP level $(\mathrm{P}<0.001)$, and intrahepatic metastasis $(\mathrm{P}=0.004)$ were found to be significant independent risk factors for recurrence after curative hepatectomy (Table IV). Fig. 3 shows the overall cumulative recurrence rate curve according to pre- and postoperative AFP level. When limited to the patients with a positive AFP level before operation, 52 patients whose AFP value remained positive after operation had significantly higher recurrence rate than 31 whose value changed to negative $(\mathrm{P}<0.001)$. Among the patients with a positive minimum postoperative AFP level, 70.4\% (38/54) experienced recurrence within 1 postoperative year, $22.2 \%$ $(12 / 54)$ after this time period and only $7.4 \%$ (4/54) did not experience recurrence until the end of follow-up.

Correlation between AFP level and hepatitis. To evaluate the correlation between AFP level and grade of hepatitis activity, we compared minimum postoperative AFP level with simultaneous alanine aminotransferase (ALT) level. Univariate 
Table III. Sensitivity, specificity, positive predictive value (PPV) and negative predictive value (NPV) of minimum postoperative $\alpha$-fetoprotein (AFP), des- $\gamma$-carboxy prothrombin (DCP), and combination of both for detection of hepatocellular carcinoma (HCC) recurrence.

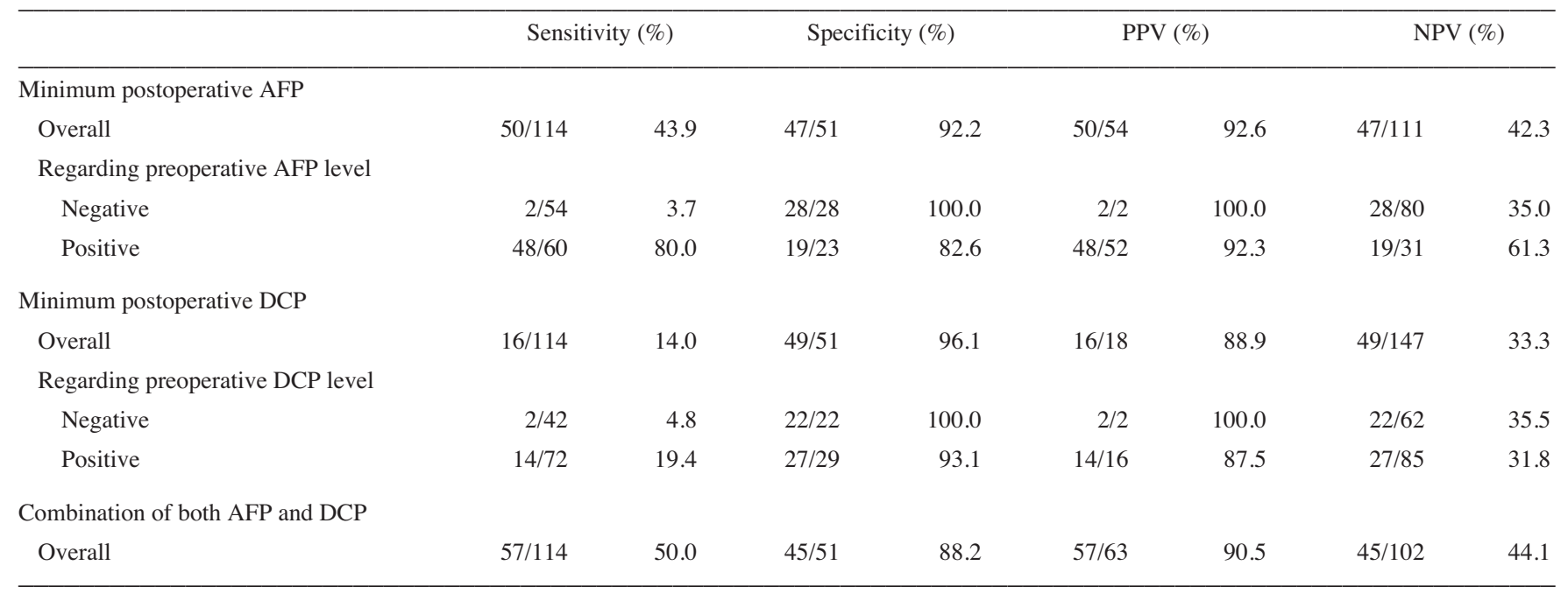

Table IV. Cumulative recurrence rate stratified by variables using Kaplan-Meier method.

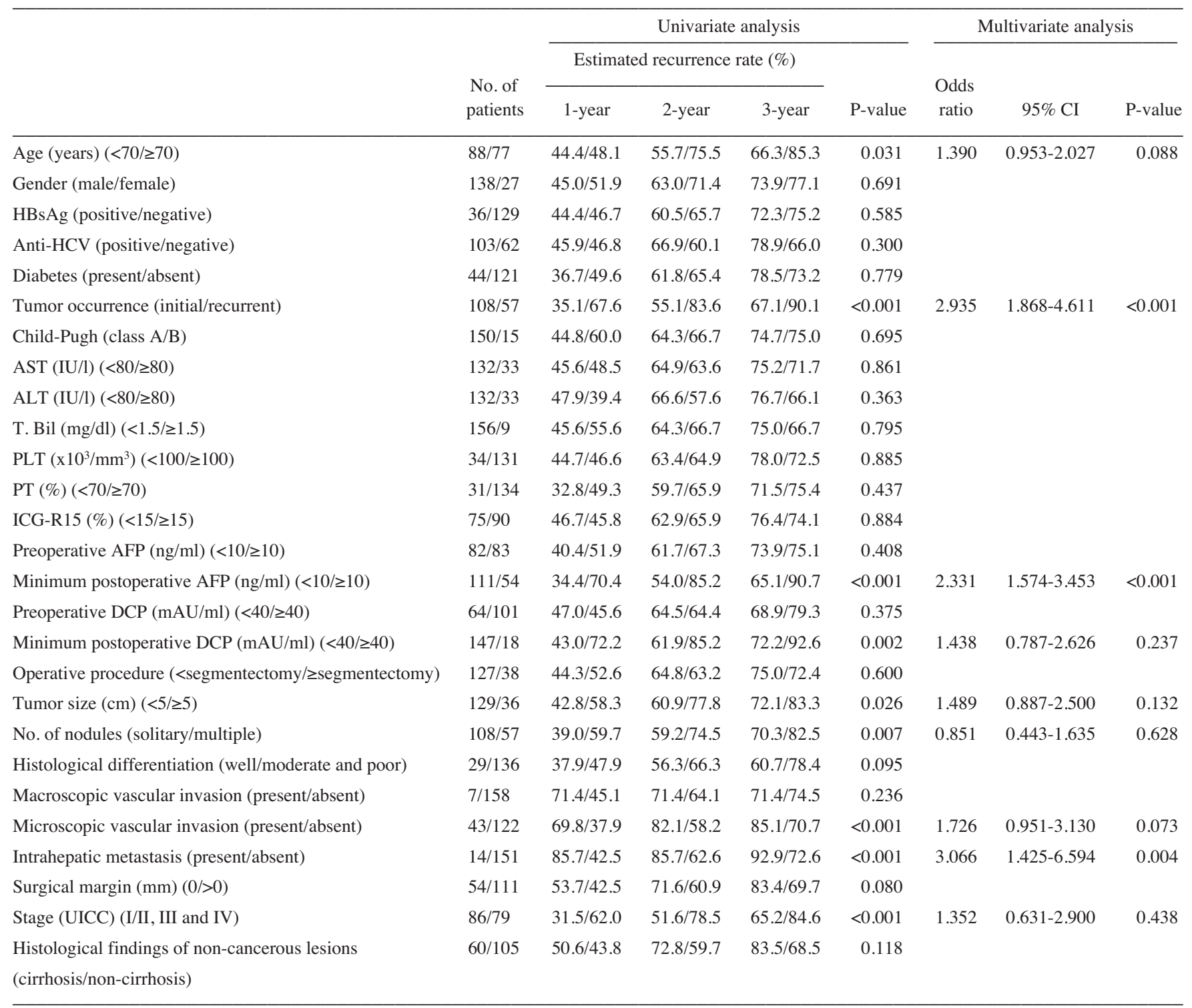




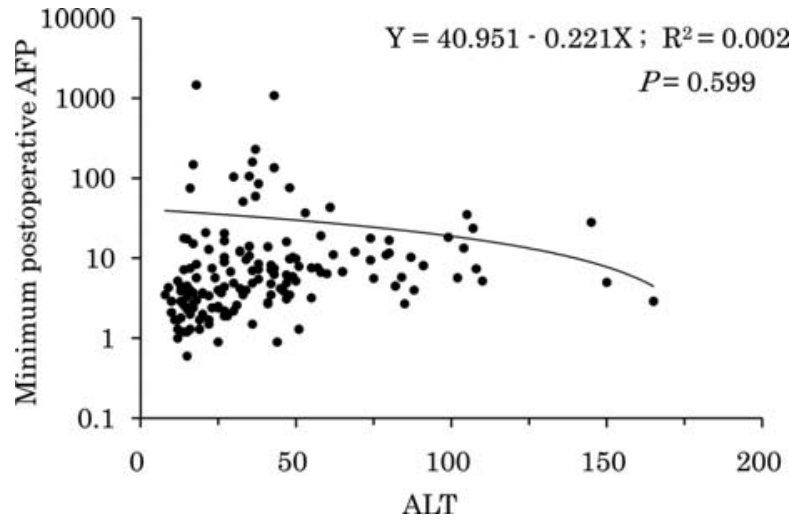

Figure 4. Correlation between minimum postoperative $\alpha$-fetoprotein (AFP) and alanine aminotransferase (ALT) levels. There was no significant correlation $(\mathrm{P}=0.599)$.
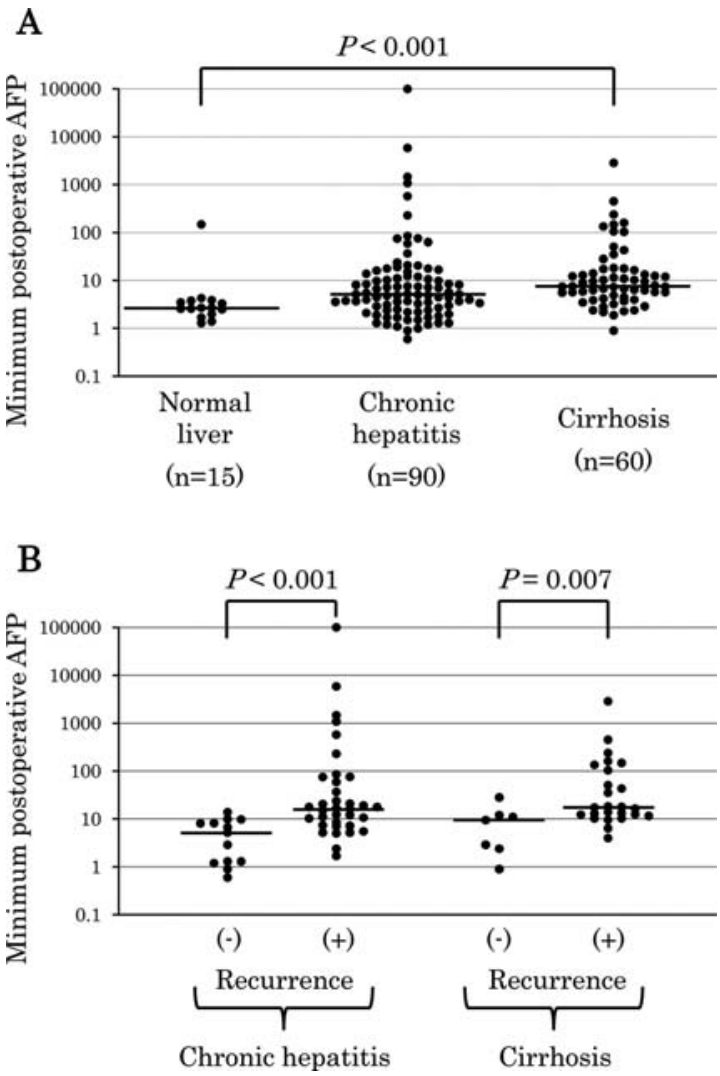

Figure 5. Correlation between minimum postoperative $\alpha$-fetoprotein (AFP) and histological findings of non-cancerous lesions. (A) Patients were divided into three groups according to grade of underlying chronic liver disease. As underlying liver disease became more severe, minimum postoperative AFP level became greater $(\mathrm{P}<0.001)$. (B) Furthermore, patients limited to those with a positive preoperative AFP level in each group were divided into two subgroups - with and without recurrence. Minimum postoperative AFP level in patients with recurrence exceeded that in patients without recurrence in the chronic hepatitis group $(\mathrm{P}<0.001)$ and cirrhosis group $(\mathrm{P}=0.007)$. Bars are medians.

regression analysis showed no statistically significant correlation ( $\mathrm{P}=0.599)$ (Fig. 4). To evaluate the correlation between AFP level and grade of underlying chronic liver disease, we compared minimum postoperative AFP level with histological findings of non-cancerous lesions. Fig. 5A shows minimum postoperative AFP level of the patients divided into three groups according to underlying chronic liver disease. As chronic liver disease became more severe, minimum postoperative AFP level became greater, and the difference was statistically significant by Kruskal-Wallis rank test $(\mathrm{P}<0.001)$. Furthermore, when patients were limited to those with a positive preoperative AFP level and the patients in each group were divided into two subgroups - those with and without recurrence, minimum postoperative AFP level of the patients with recurrence exceeded that of the patients without recurrence in the chronic hepatitis group $(\mathrm{P}<0.001)$ and cirrhosis group $(\mathrm{P}=0.007)$ (Fig. 5B).

\section{Discussion}

In this study, we demonstrated that postoperative AFP level is a useful tool for predicting HCC recurrence after curative hepatectomy. The evidence for this is that most of the patients who experienced recurrence later did not show a negative change in AFP level after curative resection. Moreover, minimum postoperative AFP level was a significant independent risk factor for recurrence. On the other hand, most of the patients who experienced recurrence later as well as those who never experienced recurrence showed a negative change in DCP level after operation, and minimum postoperative DCP was not a significant risk factor in multivariate analysis. There was no statistically significant correlation between AFP level and grade of hepatitis activity, and thus a positive level of AFP after operation might suggest a site of residual viable cancer.

Imaging modalities, including US, dynamic CT and dynamic MRI, are the gold standard for diagnosis of HCC. However, in general, since they can only detect a cancer site greater than approximately $1 \mathrm{~cm}$ in diameter, smaller cancer sites are missed before operation. Although intraoperative US is used to try to detect other cancer sites that have not been detected before operation, the limitations of US include its operator dependence and its poor ability to differentiate early HCC from dysplastic nodules in the cirrhotic liver. Therefore a positive level of AFP after operation might suggest a viable residual cancer site that has been undetectable by imaging modalities.

One reported problem of AFP and DCP is low sensitivity $(15,16)$. Although measurement of two tumor markers is recommended (17-19), the sensitivity for small HCC is not yet satisfactory. However, this study showed that, when classified by preoperative level, the sensitivity of minimum postoperative AFP level was high $(80.0 \%)$, whereas that of minimum postoperative DCP level was still low (19.4\%). This is because AFP is superior to DCP for the diagnosis of small HCC (20). Another reported problem of AFP is low specificity because of a high false-positive rate with benign conditions such as acute and chronic active hepatitis (21-24). Several authors have demonstrated that Lens culinaris agglutinin-reactive $\alpha$-fetoprotein (AFP-L3) can distinguish between HCC and hepatitis by detecting a sugar chain microheterogeneity (25-27). Our previous studies have demonstrated that glypican-3 (GPC3) is a novel tumor marker of $\mathrm{HCC}$ and is especially useful in the early stages because of its high sensitivity (28-31). 
A limitation of our study is that it is difficult to determine whether an elevation of AFP is due to a residual cancer site or active hepatitis. However, our results showed no statistically significant correlation between levels of AFP and ALT, which is a well known marker of hepatitis activity $(32,33)$. Although the histological findings of non-cancerous lesions showed a statistically significant correlation with AFP level, our results showed that patients who had higher postoperative AFP levels were most likely to experience recurrence. Moreover, not the grade of underlying chronic liver disease but postoperative AFP level was a significant risk factor for recurrence by univariate and multivariate analyses. Therefore, in most cases, a positive level of AFP after operation might mean a residual viable cancer site and not liver cirrhosis.

Generally, two different mechanisms are responsible for HCC recurrence (34). One is recurrence due to metastasis, originating from cancer cell dissemination from the primary tumor. The other is multicentric carcinogenesis of a new tumor based on underlying hepatitis or cirrhosis. However, they are not easily distinguishable (35). Instead, we distinguished between recurrence within 1 postoperative year and that after this time period as described previously (36). The result was that the majority of patients whose postoperative AFP level remained positive experienced recurrence within 1 postoperative year. Therefore, a positive level of AFP after operation suggests a site of residual viable cancer that has already occurred before operation.

In order to prevent $\mathrm{HCC}$ recurrence from a viable but undetectable cancer site, establishment of effective adjuvant therapy is urgently needed. We have just started a phase II clinical trial of GPC3-derived peptide vaccine for adjuvant therapy after curative operation or ablation. GPC3 is an ideal target for anticancer immunotherapy because its expression is detected specifically in most HCCs even in the early stages and is correlated with a poor outcome (37-41).

In conclusion, we have shown that minimum postoperative AFP level is an important risk factor for recurrence after curative hepatectomy. A positive level of AFP after operation might suggest a residual viable cancer site. The need for effective adjuvant therapy and close follow-up is suggested in patients with a positive postoperative AFP level. In addition, further studies will be needed to find novel useful serum markers that have better sensitivity for early detection of $\mathrm{HCC}$ recurrence.

\section{Acknowledgements}

This work was supported by Health and Labor Sciences Research Grants for Research on hepatitis from the Ministry of Health, Labor and Welfare.

\section{References}

1. Parkin DM: Global cancer statistics in the year 2000. Lancet Oncol 2: 533-543, 2001

2. McGlynn KA, Tsao L, Hsing AW, Devesa SS and Fraumeni JF Jr: International trends and patterns of primary liver cancer. Int $\mathrm{J}$ Cancer 94: 290-296, 2001.

3. El-Serag HB and Rudolph KL: Hepatocellular carcinoma: epidemiology and molecular carcinogenesis. Gastroenterology 132: 2557-2576, 2007

4. El-Serag HB: Epidemiology of hepatocellular carcinoma in USA. Hepatol Res 37 (Suppl. 2): S88-S94, 2007.
5. Ishii $\mathrm{H}$, Yamamoto $\mathrm{J}$ and Ikari T: Adjuvant treatments for resectable hepatocellular carcinoma. J Hepatobiliary Pancreat Surg 15: 459-462, 2008.

6. Kudo M: Hepatocellular carcinoma 2009 and beyond: from the surveillance to molecular targeted therapy. Oncology 75 (Suppl. 1): S1-S12, 2008.

7. Nomura F, Ohnishi K and Tanabe Y: Clinical features and prognosis of hepatocellular carcinoma with reference to serum alpha-fetoprotein levels. Analysis of 606 patients. Cancer 64: $1700-1707,1989$

8. Shimada M, Takenaka K, Fujiwara Y, et al: Des-gammacarboxy prothrombin and alpha-fetoprotein positive status as a new prognostic indicator after hepatic resection for hepatocellular carcinoma. Cancer 78: 2094-2100, 1996.

9. Toyoda H, Kumada T, Osaki Y, et al: Staging hepatocellular carcinoma by a novel scoring system (BALAD score) based on serum markers. Clin Gastroenterol Hepatol 4: 1528-1536, 2006.

10. Carr BI, Kanke F, Wise M and Satomura S: Clinical evaluation of Lens culinaris agglutinin-reactive alpha-fetoprotein and desgamma-carboxy prothrombin in histologically proven hepatocellular carcinoma in the United States. Dig Dis Sci 52: 776-782, 2007.

11. Cucchetti A, Piscaglia F, Caturelli E, et al: Comparison of recurrence of hepatocellular carcinoma after resection in patients with cirrhosis to its occurrence in a surveilled cirrhotic population. Ann Surg Oncol 16: 413-422, 2009

12. Honda H, Ochiai K, Adachi E, et al: Hepatocellular carcinoma: correlation of $\mathrm{CT}$, angiographic and histopathologic findings. Radiology 189: 857-862, 1993.

13. Takigawa Y, Sugawara Y, Yamamoto J, et al: New lesions detected by intraoperative ultrasound during liver resection for hepatocellular carcinoma. Ultrasound Med Biol 27: 151-156, 2001.

14. Sobin LH and Wittekind CH: UICC: TNM classification of malignant tumors. 6th edition. Wiley-Liss, New York, 2002.

15. Fujiyama S, Izuno K, Gohshi K, Shibata J and Sato T: Clinical usefulness of des-gamma-carboxy prothrombin assay in early diagnosis of hepatocellular carcinoma. Dig Dis Sci 36: 1787-1792, 1991.

16. Sherman M: Surveillance for hepatocellular carcinoma. Semin Oncol 28: 450-459, 2001

17. Izuno K, Fujiyama S, Yamasaki K, Sato M and Sato T: Early detection of hepatocellular carcinoma associated with cirrhosis by combined assay of des-gamma-carboxy prothrombin and alpha-fetoprotein: a prospective study. Hepatogastroenterology 42: 387-393, 1995.

18. Toyoda H, Kumada T, Kiriyama S, et al: Prognostic significance of simultaneous measurement of three tumor markers in patients with hepatocellular carcinoma. Clin Gastroenterol Hepatol 4: 111-117, 2006.

19. Makuuchi M, Kokudo N, Arii S, et al: Development of evidence-based clinical guidelines for the diagnosis and treatment of hepatocellular carcinoma in Japan. Hepatol Res 38: 37-51,2008.

20. Nakamura S, Nouso K, Sakaguchi K, et al: Sensitivity and specificity of des-gamma-carboxy prothrombin for diagnosis of patients with hepatocellular carcinomas varies according to tumor size. Am J Gastroenterol 101: 2038-2043, 2006.

21. Kew M: Alpha-fetoprotein in primary liver cancer and other diseases. Gut 15: 814-821, 1974.

22. Bloomer JR, Waldmann TA, McIntire KR and Klatskin G: Alpha-fetoprotein in non-neoplastic hepatic disorders. JAMA 233: 38-41, 1975.

23. Alpert E and Feller ER: Alpha-fetoprotein (AFP) in benign liver disease. Evidence that normal liver regeneration does not induce AFP synthesis. Gastroenterology 74: 856-858, 1978.

24. Bayati N, Silverman AL and Gordon SC: Serum alpha-fetoprotein levels and liver histology in patients with chronic hepatitis C. Am J Gastroenterol 93: 2452-2456, 1998.

25. Okuda K, Tanaka M, Kanazawa N, et al: Evaluation of curability and prediction of prognosis after surgical treatment for hepatocellular carcinoma by lens culinaris agglutinin-reactive alpha-fetoprotein. Int J Oncol 14: 265-271, 1999.

26. Hayashi K, Kumada T, Nakano S, et al: Usefulness of measurement of Lens culinaris agglutinin-reactive fraction of alphafetoprotein as a marker of prognosis and recurrence of small hepatocellular carcinoma. Am J Gastroenterol 94: 3028-3033, 1999. 
27. Tateishi R, Shiina S, Yoshida H, et al: Prediction of recurrence of hepatocellular carcinoma after curative ablation using three tumor markers. Hepatology 44: 1518-1527, 2006.

28. Nakatsura T, Yoshitake Y, Senju S, et al: Glypican-3, overexpressed specifically in human hepatocellular carcinoma, is a novel tumor marker. Biochem Biophys Res Commun 306: 16-25, 2003.

29. Nakatsura $T$ and Nishimura Y: Usefulness of the novel oncofetal antigen glypican-3 for diagnosis of hepatocellular carcinoma and melanoma. BioDrugs 19: 71-77, 2005.

30. Shirakawa H, Kuronuma T, Nishimura Y, et al: Glypican-3 is a useful diagnostic marker for a component of hepatocellular carcinoma in human liver cancer. Int J Oncol 34: 649-656, 2009.

31. Hayashi E, Motomura Y, Shirakawa H, et al: Detection of glypican-3-specific CTLs in chronic hepatitis and liver cirrhosis. Oncol Rep 22: 149-154, 2009.

32. Tarao K, Takemiya S, Tamai S, et al: Relationship between the recurrence of hepatocellular carcinoma (HCC) and serum alanine aminotransferase levels in hepatectomized patients with hepatitis C virus-associated cirrhosis and HCC. Cancer 79: 688-694, 1997.

33. Goldstein NS, Blue DE, Hankin R, et al: Serum alphafetoprotein levels in patients with chronic hepatitis C. Relationships with serum alanine aminotransferase values, histologic activity index, and hepatocyte MIB-1 scores. Am J Clin Pathol 111: 811-816, 1999.

34. Nakashima O and Kojiro M: Recurrence of hepatocellular carcinoma: multicentric occurrence or intrahepatic metastasis? A viewpoint in terms of pathology. J Hepatobiliary Pancreat Surg 8: 404-409, 2001.
35. Imamura H, Matsuyama Y, Tanaka E, et al: Risk factors contributing to early and late phase intrahepatic recurrence of hepatocellular carcinoma after hepatectomy. J Hepatol 38: 200-207, 2003

36. Choi GH, Kim DH, Kang CM, et al: Prognostic factors and optimal treatment strategy for intrahepatic nodular recurrence after curative resection of hepatocellular carcinoma. Ann Surg Oncol 15: 618-629, 2008.

37. Nakatsura T, Komori H, Kubo T, et al: Mouse homologue of a novel human oncofetal antigen, glypican-3, evokes T-cellmediated tumor rejection without autoimmune reactions in mice. Clin Cancer Res 10: 8630-8640, 2004.

38. Motomura Y, Senju S, Nakatsura T, et al: Embryonic stem cellderived dendritic cells expressing glypican-3, a recently identified oncofetal antigen, induce protective immunity against highly metastatic mouse melanoma, B16-F10. Cancer Res 66: 2414-2422, 2006.

39. Komori H, Nakatsura T, Senju S, et al: Identification of HLA-A2or HLA-A24-restricted CTL epitopes possibly useful for glypican-3-specific immunotherapy of hepatocellular carcinoma. Clin Cancer Res 12: 2689-2697, 2006.

40. Motomura Y, Ikuta Y, Kuronuma T, et al: HLA-A2 and -A24restricted glypican-3-derived peptide vaccine induce specific CTLs: preclinical study using mice. Int J Oncol 32: 985-990, 2008.

41. Shirakawa H, Suzuki H, Shimomura M, et al: Glypican-3 expression is correlated with poor prognosis in hepatocellular carcinoma. Cancer Sci 100: 1403-1407, 2009. 